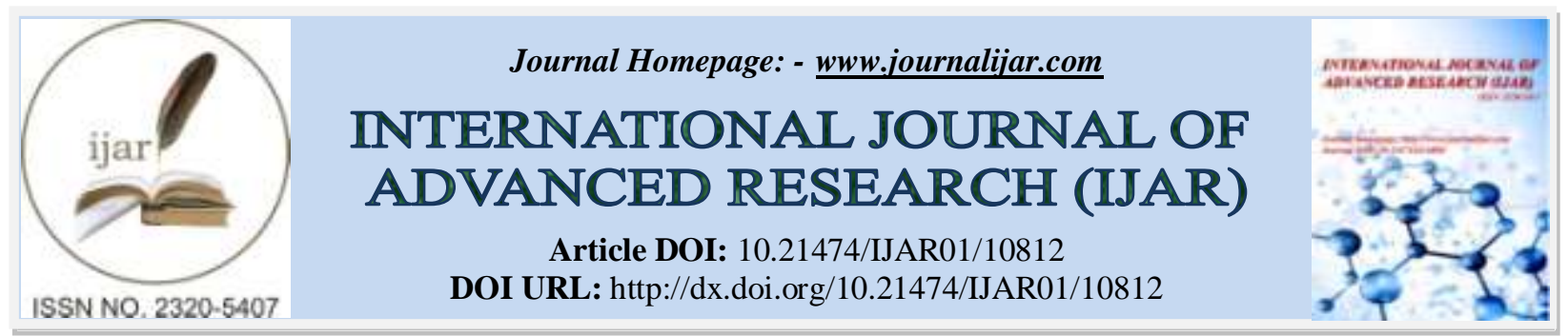

RESEARCH ARTICLE

\title{
COMPARATIVE EVALUATION OF ROOT CANAL FILLING QUALITY OF NEWLY DEVELOPED ENDODONTIC SEALERS APPLIED USING GUTTA-PERCHA CONE MEDIATED ULTRASONIC ACTIVATION: AN IN-VITRO STUDY
}

\section{Yogesh Damade, Sunanda Gaddalay, Ramchandra Kabir, Sharvaree Deshpande, Anita Kale and Deepak Nagargoje}

Department of Conservative Dentistry and Endodontics, MIDSR Dental College and Hospital, Latur, Maharashtra, India. Pin code -413512 .

\section{Manuscript Info}

\section{Manuscript History}

Received: 12 February 2020

Final Accepted: 14 March 2020

Published: April 2020

Key words:-

Bioceramic Sealer, Endoseal Mta, Guttaflow Bioseal, Pozzolan-Based Mta Sealer, Root Canal Filling Quality, Ultrasonic Activation

\section{Abstract}

Introduction: The aim of this study is to evaluate the root canal filling quality of newly developed endodontic sealers applied using Guttapercha cone mediated ultrasonic activation.

Materials and Methods: 50 freshly extracted mandibular premolars were selected. After access opening and instrumentation, root canal filling was carried out using, Endoseal MTA (EMS), Guttaflow BioSeal (GBS), and AH plus, with or without ultrasonic activation. Samples were sliced into coronal, middle and apical sections, perpendicular to long axis of the tooth and observed under a digital stereomicroscope for filling quality assessment, using a void score system. Data were analyzed by one way ANOVA and post hoc tests.

Results: Apical thirds showed more voids compared to middle and coronal thirds in all five groups $(\mathrm{p}<0.05)$. Least voids were found in $\mathrm{AH}$ plus group using warm vertical compaction (APW) which was statistically significant $(\mathrm{p}<0.05)$. When EMS was used alone, it has higher voids compared to APW ( $\mathrm{p}<0.05$ ), but is as effective as APW when used along with ultrasonic activation. Ultrasonic activation has shown no effect on lowering void score in between EMS and GBS groups ( $>0.05)$. APW group performed better than EBS and GBS group.

Conclusion: Endoseal MTA is a potential sealing material with better void sealing ability comparable to AH Plus, when used under ultrasonic activation.

Copy Right, IJAR, 2020,. All rights reserved.

\section{Introduction:-}

The success of a root canal treatment is largely dependent on a combination of proper instrumentation, irrigation and obturation of the complex root canal system without voids. Obturation not only seals the apex from periapical tissue fluids but also prevents bacterial contamination, leakage and seepage of any remaining irritants into the canal. However, an impervious seal cannot be achieved with the available materials and techniques used for obturation and some amount of leakage may occur (Kim JC et al, 2020). Root canal failures occur mainly due to the presence of voids in the obturated canals which act as a dead space for growth of microorganisms (Moazami $F$ et al, 2020). Gutta percha based materials have been widely used for obturation worldwide. But it acts only as a filling material 
and does not adhere to canal wall dentin. Therefore it is necessary to use a sealer to increase the sealing ability of gutta percha. Several root canal sealers are currently available. Endodontic sealers are used in order to achieve a fluid-tight seal between the dentinal wall and core filling material. One such sealer is Mineral trioxide aggregate (MTA).

MTA is composed of calcium, silica, and bismuth. it has been widely used in variety of applications such as rootend filling, perforation repair, or apical/coronal sealing material during regenerative endod ontic procedures, pulp capping, pulpotomy, apical barrier formation in teeth with open apexes (Parirokh M et al,2010). It has a long setting time, high $\mathrm{pH}$, and low compressive strength. It possesses some antibacterial and antifungal properties, depending on its powder-to-liquid ratio (Parirokh $m$ et al 2010). As MTA is hydraulic hydrophilic, it can be used to reduce fluid flow rate and seal the apex in teeth with wide open apices (Prati C et al, 2014; Kim H et al, 2016) and can use the natural moisture in the root canal, (Kim J et al, 2020). Such characteristic features of mta appear to be important in biomineralization of dentinal tubules for enhanced sealing of the root canal system making it a good candidate for root canal filling (Yoo Y et al, 2016). However, it cannot be recommended as a routine sealer or root canal filling material because of its disadvantages such as difficulty in filling of complicated root canal system, long setting time (Lee J et al, 2017), (Yazdi K et al, 2019), sandy property and irretrievability of the material in retreatment cases (Kim J et al, 2020).

A recently developed variant of MTA is the Endoseal MTA (Maruchi, Wonju, Korea). It is a pozzolan-based MTA which is premixed and preloaded in an air-tight syringe, and can be applied directly into the root canals. (Moazami F et al, 2020). It is composed of calcium silicates, calcium aluminates, calcium aluminoferrite and calcium sulfates. The main component of this sealer, the pozzolan cement, gets its cementitious properties after pozzolanic reaction which includes calcium hydroxide and water, and enables sufficient flow of the pre-mixed substrate though injection tips with adequate working consistency. (Yoo Y et al, 2016). Yazdi K et al (2019), reported that warm vertical compaction and single cone technique with Endoseal MTA show significantly lower percentage of voids ( $\mathrm{P}$ value

$<0.05)$ compared to that in the GuttaFlow2 and Endosequence BC sealer groups, when artificial resorption cavities were filled. Few studies reported that Endoseal MTA had a better performance with gutta-percha cone-mediated ultrasonic activation (Moazami F et al, 2020).

Another new root canal sealer containing calcium silicate is GuttaFlow Bioseal (Coltène, Switzer land), also known as GuttaFlow 3. It is composed of gutta-percha, polydimethylsiloxane, zirconium oxide, platinum, and bioactive ceramic glass. GuttaFlow Bioseal was developed to improve the bioactivity of GuttaFlow (Reszka P, 2019). It has the same formulation as the GuttaFlow sealer but also includes calcium silicate, which, upon contact with biological tissues, releases natural repair constituents and aids in the regeneration of periapical tissues. It provides repair along with obturation (Camargo RVD et al, 2017; Reszka P et al, 2019). Complementary analysis by means of atomic absorption spectrometry showed release of sodium ions from GuttaFlow Bioseal. This is due to the addition of bioactive glass ceramics. Calcium and sodium ion released, increase the $\mathrm{pH}$ of the dental environment and prohibits bacterial viability (Camargo RVD et al, 2017). Hoikkala N-PJ et al (2018) reported that GuttaFlow BioSeal exhibited significantly higher water sorption and solubility in comparison with Guttaflow 2. The exposed particles of bioglass ceramic particles release ions into the surrounding media, thereby increasing the $\mathrm{pH}$ to 7.9 which causes antimicrobial effects. Biomineralization occurs on the surface of the sealer suggesting that the precipitation is hydroxyapatite (Hoikkala N-PJ et al, 2018). Histopathologic evaluation revealed that GuttaFlow sealers were more cytocompatible than AH Plus and MTA Fillapex, while GuttaFlow Bioseal favored cementoblast differentiation of human periodontal stem cells in the absence of any growth factors (Rodríguez-Lozano F et al, 2019).

Through the development of new endodontic sealers and tests, evaluation and knowledge about them may be enhanced. The aim of this study was to evaluate the root canal filling quality of two recently developed endodontic sealers Endoseal MTA (Maruchi, Korea) and GuttaFlow Bioseal (Coltene, Switzerland) in comparison with AH Plus, using Gutta-percha cone mediated ultrasonic activation.

\section{Materials And Methods:-}

Sample of fifty human single-rooted freshly extracted mandibular premolars were selected. Access cavity was prepared and a size $10 \mathrm{~K}$-file was used to determine the working length $0.5 \mathrm{~mm}$ short of the radiographic apex. The root canal cleaning and shaping were done with Protaper rotary files till F3 preparation. Canals were irrigated with 5 $\mathrm{ml}$ of $5.25 \%$ sodium hypochlorite, $5 \mathrm{ml}$ of $17 \%$ EDTA and followed by normal saline. Root canals were dried with 
absorbent paper points. At the end of the preparation, the samples were randomly divided into five groups, with 10 specimens each. The five different protocols used were:

Group A: EMS (Endoseal MTA + single cone without ultrasonic activation)

Group B: EMSU (Endoseal MTA + single cone with ultrasonic activation)

Group C: GBS (GuttaFlow Bioseal + single cone without ultrasonic activation)

Group D: GBSU (GuttaFlow Bioseal + single cone with ultrasonic activation)

Group E: APW (AH plus + warm vertical compaction)

The files were frequently cleaned to remove debris from their flutes, and the irrigating solutions were frequently replaced to maintain effectiveness. During obturation, tug back was checked at the working length and confirmed radiologically. The EMS and GBS sealers which come in pre-mixed syringes with injection tips were inserted into the canals. The root canals were completely covered by the sealer. In the groups of ultrasonically activated sealers (EMSU, GBSU), activation was performed immediately after sealer placement using an ultrasonic tip attached to an ultrasonic device. In EMS, EMSU, GBS, GBSU groups a single cone was inserted upto the working length. In APW group, the AH Plus sealer (Dentsply, Maillefer, North America) was manipulated according the manufacturer's instructions and inserted into the root canals followed by warm vertical compaction of gutta percha. After obturation, all experimental samples were stored at $37 \circ \mathrm{C}$ and $100 \%$ humidity for 24 hours to allow the sealers to set. Later, they were sectioned perpendicular to the longitudinal axis of root at $4 \mathrm{~mm}$ of distance at the level of coronal, middle and apical third using a slow speed diamond wheel.

Each assigned slice was observed under Digital Stereomicroscope (Nikon eclipse E200 Japan) under a magnification of 40x. Digital image of each segment was taken and were observed to estimate the number of voids. Filling quality (number of voids) was assessed by a single blinded and caliberated examiner. Numbers of voids present were counted under a scoring system of each section and the mean void scores were calculated (Kim J-A et al, 2018).

Scoring criteria for evaluating the filling quality of various sealers

1. Score 0: No voids.

2. Score 1: Well-condensed filling that showed only a few, minor air bubbles less the (less than $0.1 \mathrm{~mm}$ in diameter).

3. Score 2: An imperfectly condensed filling that showed some minor air bubbles (more than 3 defects) or medium sized air bubbles $(0.1 \mathrm{~mm}$ to $0.2 \mathrm{~mm}$ in diameter).

4. Score 3: Inadequately condensed filling that showed many minor air bubbles (more than 5 defects) or large air bubbles (more than $0.2 \mathrm{~mm}$ in diameter).

5. Score 4: Poorly condensed filling that showed many minor air bubbles (more than 7 defects) or empty space connecting separate canal walls.

Statistical analysis was performed (with SPSS software) and descriptive statistics were calculated. Intergroup comparison was done using one way ANOVA at coronal, middle and apical thirds. Post hoc tests were done to compare mean difference between individual groups. $\mathrm{P}$ value of $<0.05$ was considered to be statistically significant.

\section{Results:-}

Table 1, 2 and 3 show the mean void scores among the various groups in the coronal, middle and apical thirds respectively. Least mean void score was found in Group E (APW), followed by Group B (EMSU) in the coronal, middle and apical thirds, which was found to be highly statistically significant $(\mathrm{p}=0.0001)$. Maximum mean void score was found in the Group C (GBS), followed by Group D (GBSU).

Post hoc Tukey's test showed the mean difference between EMS and APW, GBS and APW, GBSU and APW, and EMSU and GBS groups to be statistically significant $(\mathrm{p}<0.05)$ at the coronal thirds. Significant difference was found between EMSU and GBS, EMSU and GBSU, GBS and APW, and GBSU and APW in middle thirds and between EMS and APW, GBS and APW, EMSU and GBS, EMSU and GBSU, and GBSU and APW groups in the apical third $(\mathrm{p}<0.05)$. APW showed lesser voids compared to EMS and GBS $(\mathrm{P}<0.05)$.

In coronal third, no statistically significant difference was found between EMS and EMSU, EMS and GBS, GBS and GBSU, EMSU and GBSU, EMSU and APW. It indicates that EMSU may be as effective as APW in the coronal third

$(\mathrm{p}>0.05)$. 
Similarly, there is no statistically significant difference between EMS and EMSU, EMS and GBS, EMS and APW, EMSU and APW, GBS and GBSU, in the middle third, indicating that EMS is as effective as GBS and APW with or without ultrasonic activation ( $p>0.05)$..

In the apical third, no statistically significant difference was found between EMS and EMSU, EMS and GBS, EMSU and APW, GBS and GBSU ( $p>0.05$ ). This indicates that when EMS is used alone, it has higher voids compared to APW, but is as effective as APW when used along with ultrasonic. Since there is no statistically significant difference between EMSU and APW at all three levels (coronal, middle, apical), it implies that EMS is as effective as APW under ultrasonic activation. Ultrasonic activation has shown no effect on lowering void score in between EMS and EMSU and GBS and GBSU groups ( $\mathrm{p}>0.05)$.

Table I:- Mean void scores among the five groups in the coronal third.

\begin{tabular}{|l|l|l|l|l|l|}
\hline & N & Mean & Std. Deviation & F & P value \\
\hline EMS & 10 & 2.4 & 0.97 & \multirow{2}{*}{0.970} & \\
\cline { 1 - 3 } EMSU & 10 & 1.8 & 0.42 & \\
\cline { 1 - 3 } GBS & 10 & 2.9 & 0.57 & \\
\cline { 1 - 3 } & 10 & 2.5 & 0.53 & \\
\cline { 1 - 4 } TPW & 10 & 1.4 & 0.52 & \\
\end{tabular}

*statistically significant

Table II:- Mean void scores among the five groups in the middle third.

\begin{tabular}{|l|l|l|l|l|l|}
\hline & N & Mean & Std. Deviation & F & P value \\
\hline EMS & 10 & 1.9 & 0.74 & \multirow{2}{*}{$0.70001^{*}$} \\
\cline { 1 - 4 } EMSU & 10 & 1.4 & 0.52 & & \\
\cline { 1 - 3 } GBSU & 10 & 2.7 & 0.82 & & \\
\cline { 1 - 3 } APW & 10 & 2.4 & 0.52 & & \\
\cline { 1 - 3 } Total & 10 & 1.1 & 0.57 & & \\
\hline
\end{tabular}

*statistically significant

Table III:- Mean void scores among the five groups in the apical third.

\begin{tabular}{|c|c|c|c|c|c|}
\hline & $\mathbf{N}$ & Mean & Std. Deviation & $\mathbf{F}$ & P value \\
\hline EMS & 10 & 1.8 & 0.79 & \multirow[t]{6}{*}{13.64} & \multirow[t]{6}{*}{$0.0001^{*}$} \\
\hline EMSU & 10 & 1.3 & 0.48 & & \\
\hline GBS & 10 & 2.5 & 0.71 & & \\
\hline GBSU & 10 & 2.2 & 0.42 & & \\
\hline$\overline{\text { APW }}$ & 10 & 0.8 & 0.42 & & \\
\hline Total & 50 & 1.72 & 0.83 & & \\
\hline
\end{tabular}

*statistically significant

Figure 1:- Bar diagram showing mean void scores in the coronal, middle and apical thirds among the five groups.

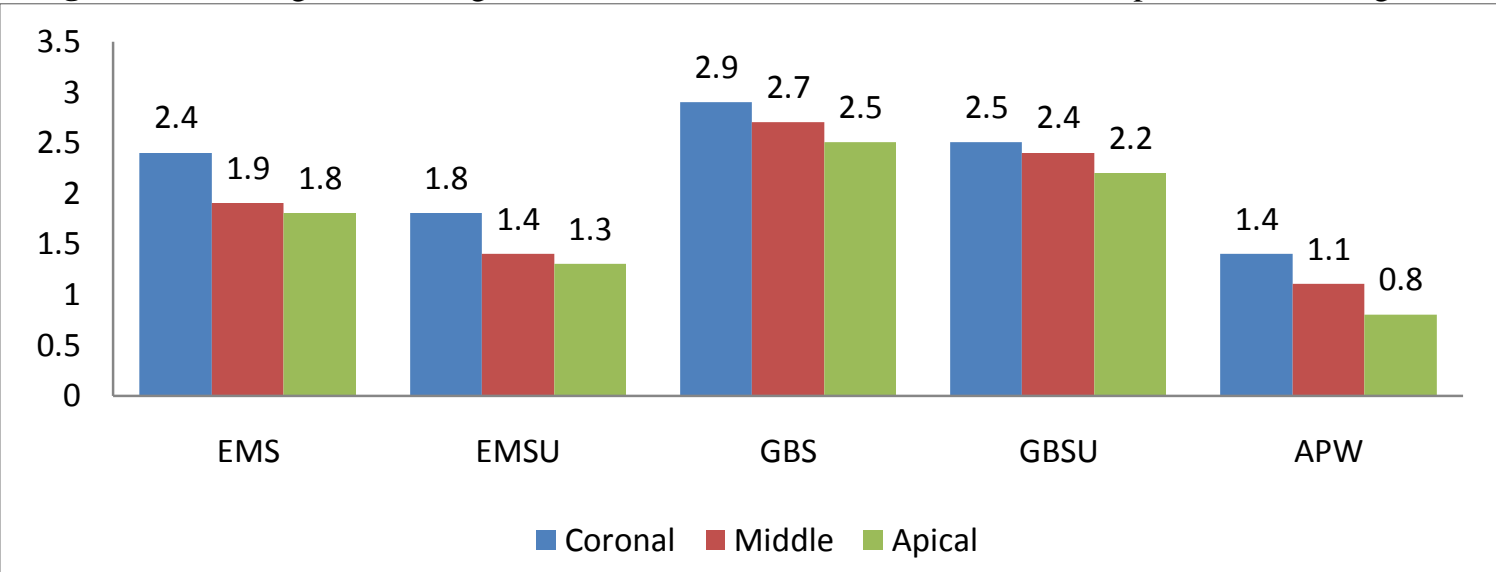


Figure 1 shows the mean void scores among the five treatment groups. Apical third showed better sealing of voids and better condensation compared to middle and coronal thirds across all the groups.

\section{Discussion:-}

Bio-ceramics are amongst the recently introduced materials in endodontics. They are biocompatible ceramic materials or metal oxides with enhanced sealing ability, and antibacterial and antifungal properties. They achieve excellent hermetic seal, form a chemical bond with the tooth structure and have good radiopacity (Raghavendra SS et al, 2017). Dr.Torabinejad in 1993 successfully introduced a bioceramic material in endodontics known as MTA (Raghavendra SS et al, 2017). It was recommended initially as a root-end filling material and subsequently has been used for pulp capping, pulpotomy, apexogenesis, apical barrier formation in teeth with open apexes, repair of root perforations, and as a root canal filling material.

In the present study, APW showed lesser voids compared to EMS and GBS. Though there was no effect of ultrasonic activation on void scores among EMS and EMSU, EMSU showed similar voids scored as APW. These results are similar to a study done by Kim J-A et al (2018) who reported that under stereomicroscopic evaluation, the EMS group showed a higher number of voids and a higher void score compared to EMSU and APW groups (P <

.05). Endoseal MTA performed best when used along with GP cone-mediated ultrasonic activation. (Moazami $\mathrm{F}$ et al, 2020; Kim J et al, 2018). Endoseal MTA showed significantly less void percentage compared to the AH Plus Jet group $(\mathrm{p}<0.05)$ in a study conducted by Kim JC et al $(2020)$. No difference was found in the percentage of voids when Endoseal MTA was applied using single cone and continuous wave obturation methods according to Kim S et al (2017).

Lim E-S et al (2015) investigated the physical properties and biocompatibility of calcium-silicate-based root canal sealer (Endoseal) in comparison with MTA and a resin-based sealer (AH plus). Endoseal showed high alkalinity similar to that of MTA and high flow. Lee JK et al (2017) reported that EndoSeal MTA had the longest setting time than the AH-Plus $(P<0.05)$. AH-Plus and EndoSeal MTA showed statistically higher radiopacity $(P<0.05)$. Set samples of 3 epoxy resin-based sealers and EndoSeal MTA presented a significant increase of $\mathrm{pH}$ over experimental time for 4 weeks. Kim Y et al (2019) compared the penetration ability of calcium silicate root canal sealers and conventional resin-based sealer using confocal laser scanning microscopy. The maximum sealer penetration depth was low in the apical area and high in the coronal area in the AH Plus and Endoseal MTA groups. In the BioRoot RCS group, maximum sealer penetration was observed in the middle third. Hwang JH et al (2015) evaluated the bacterial leakage resistance and root canal lining efficacy of various root canal filling materials and methods by using confocal laser-scanning microscope. Group EMS showed the better sealer distribution score than the other groups and less bacterial leakage score than GuttaFlow.

Kim SR et al (2019) compared the efficiency of root canal filling procedures and the retrievability of the filling material with various sealers. EndoSeal MTA showed significantly shorter time of filling procedure. The number of voids did not show significant differences between Endoseal MTA with single cone technique and AH Plus with continuous wave technique. There were no significant differences between both groups for remnant score. Yazdi KA et al (2019), reported that warm vertical compaction and single cone technique with Endoseal MTA show significantly lower percentage of voids $(\mathrm{P}$ value $<0.05)$ compared to that in the GuttaFlow2 and Endosequence $\mathrm{BC}$ sealer groups, when artificial resorption cavities were filled. Yoo Y-J et al (2016) found that enhanced biomineralization of the dentinal tubules beyond the penetrated sealer tag was confirmed under the SEM observation when Pozzolan $(\mathrm{Pz})$ based Endoseal MTA was used with gutta percha. Thus Pz-MTA cement can be used as a promising bioactive root canal sealer to enhance biomineralization of dentinal tubules under controlled environment. Silva EJNL et al (2016) demonstrated that AH Plus had significant superior resistance to dislodgment compared with EndoSeal MTA or MTA Fillapex. EndoSeal had satisfactory bond strength performance for application in endodontic therapy compared with MTA Fillapex, and although it is not able to improve adhesion compared with AH Plus.

According to Kim $\mathrm{H}$ et al (2016), Endoseal and Endoseal MTA, new MTA products, were faster to use than AH-26, but did not seal the root canal walls or penetrate the dentinal tubules as well. Therefore, AH-26 and GP cones are recommended as root canal filling material for mature permanent teeth, while Endoseal and Endoseal MTA may be useful when treating the root canals with an open apex. Though Endoseal MTA shows satisfactory results, it has some disadvantages like discoloration due to release of ferrous ions, long setting time, working time less than 4 min, improper handling properties, inadequate compressive strength and no known solvent for MTA, which makes it difficult to remove from root canals (Rawtiya M et al, 2013). 
When Akcay $\mathrm{M}$ et al (2016) assessed the dentinal tubules penetration of various sealers, no statistically significant difference was found between AH Plus, MTA Fillapex, and GuttaFlow Bioseal. Er:YAG laser activation with photon- induced- photoacoustic streaming activation (PIPS) and Passive ultrasonic irrigation (PUI) had significantly higher penetration than conventional needle irrigation. Similarly, Lopes FC et al (2019) evaluated the effect of ultrasonic and sonic activation on physicochemical properties of AH Plus, MTA Fillapex, ADSeal, GuttaFlow Bioseal, and GuttaFlow 2 sealers. Ultrasonic and sonic activation promoted changes in the physicochemical properties of the evaluated root canal sealers, mainly in setting time and flow. This is in contrast to the present study wherein there was no added benefit of ultrasonic activation on Endoseal MTA and GuttaFlow BioSeal.

Gandolfi M et al (2016) demonstrated that GuttaFlow Bioseal showed low solubility and porosity, high water sorption, moderate calcium release, good alkalinizing activity and apatite forming ability. Hoikkala N-PJ et al (2018) reported that GuttaFlow BioSeal exhibited significantly higher water sorption and solubility in comparison with Guttaflow 2.

Bouillaguet S et al (2007) reported that Pulp canal sealer (PCS) and AH-Plus allowed significantly more fluid flow than GuttaFlow and Epiphany. Of the materials tested, GuttaFlow and Epiphany sealed the apex of straight roots most effectively. Whereas, Camargo RVD et al (2017), demonstrated that no statistically significant difference in the flow, among the sealers AH Plus, GuttaFlow 2, GuttaFlow BioSeal, and MM Seal. GuttaFlow 2 and GuttaFlow BioSeal showed highest solubility and dimensional change and higher calcium release rates than either MM Seal and AH Plus and that GuttaFlow Bioseal is the only sealer with a high sodium release rate. All the other sealers showed low rates of potassium release (Camargo RVD et al, 2017). Pedullà E et al (2019) investigated the retreatability of two calcium silicate- based materials (BioRoot RCS, Septodont, Saint-Maur- des- Fossés, France and GuttaFlow Bioseal. Significantly smaller volumes of root filling remnants of GuttaFlow Bioseal, than BioRoot RCS, were present after their removal with rotary instruments and irrigation. Collado-González M et al (2017) demonstrated that GuttaFlow Bioseal and GuttaFlow2 have lower cytotoxicity than MTA Fillapex and AH plus.

\section{Conclusion:-}

Endoseal MTA, when used along with ultrasonic activation shows promising results, which are comparable to AH plus. Without ultrasonic activation EMS and GBS groups performed poorly compared to APW group. Ultrasonic activation showed no effect on lowering void scores in the EMS and GBS groups ( $p>0.05$ ). Clinical trial tests and long term follow-up studies using various types of the sealers would be highly valuable to evaluate the sealers' clinical performances. Though the results are satisfactory, further research and scientific studies are needed to confirm them.

\section{Ethical considerations:}

The research protocol was approved by the Institutional Ethics Committee of MIDSR Dental College and Hospital, Latur, Maharashtra, India.

\section{Conflict of interests:}

The authors declare that they have no conflict of interests.

\section{References:-}

1. Akcay, M., Arslan, H., Durmus, N., Mese, M., et al. (2016) Dentinal tubule penetration of AH Plus, iRoot SP, MTA fillapex, and guttaflow bioseal root canal sealers after different final irrigation procedures: A confocal microscopic study. Lasers in Surgery and Medicine. 48 (1), 70-76. Available from: doi:10.1002/1sm.22446.

2. Bouillaguet, S., Shaw, L., Barthelemy, J., Krejci, I., et al. (2008) Long-term sealing ability of Pulp Canal Sealer, AH-Plus, GuttaFlow and Epiphany. International Endodontic Journal. 41 (3), 219-226. Available from: doi:10.1111/j.1365-2591.2007.01343.x.

3. Camargo, R.V.D., Silva-Sousa, Y.T.C., Rosa, R.P.F.D., Mazzi-Chaves, J.F., et al. (2017) Evaluation of the physicochemical properties of silicone- and epoxy resin-based root canal sealers. Brazilian Oral Research. 31 (0). Available from: doi:10.1590/1807-3107bor-2017.vol31.0072.

4. Collado-González, M., Tomás-Catalá, C.J., Oñate-Sánchez, R.E., Moraleda, J.M., et al. (2017) Cytotoxicity of GuttaFlow Bioseal, GuttaFlow2, MTA Fillapex, and AH Plus on Human Periodontal Ligament Stem Cells. Journal of Endodontics. 43 (5), 816-822. Available from: doi:10.1016/j.joen.2017.01.001.

5. Gandolfi, M., Siboni, F. \& Prati, C. (2016) Properties of a novel polysiloxane-guttapercha calcium silicatebioglass-containing root canal sealer. Dental Materials. 32 (5). Available from: doi:10.1016/j.dental.2016.03.001. 
6. Hoikkala, N.-P.J., Wang, X., Hupa, L., Smått, J.-H., et al. (2018) Dissolution and mineralization characterization of bioactive glass ceramic containing endodontic sealer Guttaflow Bioseal. Dental Materials Journal. 37 (6), 988994. Available from: doi:10.4012/dmj.2017-224.

7. Hwang, J.H., Chung, J., Na, H.-S., Park, E., et al. (2015) Comparison of bacterial leakage resistance of various root canal filling materials and methods: Confocal laser-scanning microscope study. Scanning. 37 (6), $422-428$. Available from: doi:10.1002/sca.21231.

8. Kim, H., Kim, Y., Nam, S., Taeyub, K., et al. (2016) Evaluation of Sealing Effect and Working Time of Root Canal Filling MTA Materials. The Journal Of The Korean Academy Of Pedtatric Dentistry. 43 (2), 129-136. Available from: doi:10.5933/jkapd.2016.43.2.129.

9. Kim JC, Moe MM, Kim SK. A micro-computed tomographic evaluation of root canal filling with a single guttapercha cone and calcium silicate sealer. Restor Dent Endod. 2020 Jan;45:e18. https://doi.org/10.5395/rde.2020.45.e18

10. Kim, J.-A., Hwang, Y.-C., Rosa, V., Yu, M.-K., et al. (2018) Root Canal Filling Quality of a Premixed Calcium Silicate Endodontic Sealer Applied Using Gutta-percha Cone-mediated Ultrasonic Activation. January 2018. Journal of endodontics. Available from: https://www.ncbi.nlm.nih.gov/pubmed/29102078 [Accessed: 8 April 2020].

11. Kim, S.R., Kwak, S.W., Lee, J.K., Goo, H.J., et al. (2019) Efficacy and retrievability of root canal filling using calcium silicate- based and epoxy resin- based root canal sealers with matched obturation techniques. Australian Endodontic Journal. 45 (3), 337-345. Available from: doi:10.1111/aej.12323.

12. Kim, S., Kim, S., Park, J.-W., Jung, I.-Y., et al. (2017) Comparison of the Percentage of Voids in the Canal Filling of a Calcium Silicate-Based Sealer and Gutta Percha Cones Using Two Obturation Techniques. Materials. 10 (10), 1170. Available from: doi:10.3390/ma10101170.

13. Kim, Y., Kim, B.-S., Kim, Y.-M., Lee, D., et al. (2019) The Penetration Ability of Calcium Silicate Root Canal Sealers into Dentinal Tubules Compared to Conventional Resin-Based Sealer: A Confocal Laser Scanning Microscopy Study. Materials. 12 (3), 531. Available from: doi:10.3390/ma12030531.

14. Lee, J.K., Kwak, S.W., Ha, J.-H., Lee, W., et al. (2017) Physicochemical Properties of Epoxy Resin-Based and Bioceramic-Based Root Canal Sealers. Bioinorganic Chemistry and Applications. 20171-8. Available from: doi:10.1155/2017/2582849.

15. Lim, E.-S., Park, Y.-B., Kwon, Y.-S., Shon, W.-J., et al. (2015) Physical properties and biocompatibility of an injectable calcium-silicate-based root canal sealer: in vitro and in vivo study. 21 October 2015. BMC oral health. Available from: https://www.ncbi.nlm.nih.gov/pmc/articles/PMC4618726/ [Accessed: 8 April 2020].

16. Lopes, F.C., Zangirolami, C., Mazzi-Chaves, J.F., Silva-Sousa, A.C., et al. (2019) Effect of sonic and ultrasonic activation on physicochemical properties of root canal sealers. Journal of Applied Oral Science. 27. Available from: doi:10.1590/1678-7757-2018-0556.

17. Moazami F, Naseri M, Malekzadeh P. Different Application Methods for Endoseal MTA Sealer: A Comparative Study. Iran Endod J. 2020;15(1): 44-9. Doi: 10.22037/iej.v15i1.27568.

18. Parirokh, M. \& Torabinejad, M. (2009) Mineral Trioxide Aggregate: A Comprehensive Literature Review-Part I: Chemical, Physical, and Antibacterial Properties. 7 December 2009. Journal of Endodontics. Available from: https://www.sciencedirect.com/science/article/pii/S0099239909007663 [Accessed: 8 April 2020].

19. Pedullà, E., Abiad, R.S., Conte, G., Khan, K., et al. (2019) Retreatability of two hydraulic calcium silicate- based root canal sealers using rotary instrumentation with supplementary irrigant agitation protocols: a laboratory- based micro- computed tomographic analysis. International Endodontic Journal. 52 (9), 1377-1387. Available from: doi:10.1111/iej.13132.

20. Prati, C., Siboni, F., Polimeni, A., Bossu, M., et al. (2014) Use of calcium-containing endodontic sealers as apical barrier in fluid-contaminated wide-open apices. Journal of Applied Biomaterials \& Functional Materials. 12 (3), 263-270. Available from: doi:10.5301/jabfm.5000162.

21. Raghavendra, S.S., Jadhav, G.R., Gathani, K.M. \& Kotadia, P. (2017) Bioceramics In Endodontics - A Review. Journal of Istanbul University Faculty of Dentistry. 51 (0). Available from: doi:10.17096/jiufd.63659.

22. Rawtiya, M., Verma, K., Singh, S., Munuga, S., et al. (2013) MTA-Based Root Canal Sealers. Journal of Orofacial Research. 316-21. Available from: doi:10.5005/jp-journals-10026-1057.

23. Reszka, P., Nowicka, A., Dura, W., Marek, E., et al. (2019) SEM and EDS study of TotalFill BC Sealer and GuttaFlow Bioseal root canal sealers. Dental and Medical Problems. 56 (2), 167-172. Available from: doi: $10.17219 / \mathrm{dmp} / 105561$.

24. Rodríguez-Lozano, F., Collado-González, M., Tomás-Catalá, C., García-Bernal, D., et al. (2019) GuttaFlow Bioseal promotes spontaneous differentiation of human periodontal ligament stem cells into cementoblast-like cells. Dental Materials. 35 (1), 114-124. Available from: doi:10.1016/j.dental.2018.11.003. 
25. Silva, E.J.N.L., Carvalho, N.K., Prado, M.C., Zanon, M., et al. (2016) Push-out Bond Strength of Injectable Pozzolan-based Root Canal Sealer. Journal of Endodontics. 42 (11), 1656-1659. Available from: doi:10.1016/j.joen.2016.08.009.

26. Yazdi, K.A. (2018) Comparing the Ability of Different Materials and Techniques in Filling Artificial Internal Resorption Cavities. European Endodontic Journal. Available from: doi:10.14744/eej.2018.13008.

27. Yoo, Y.-J., Baek, S.-H., Kum, K.-Y., Shon, W.-J., et al. (2015) Dynamic intratubular biomineralization following root canal obturation with pozzolan-based mineral trioxide aggregate sealer cement. Scanning. 38 (1), $50-56$. Available from: doi:10.1002/sca.21240. 